\title{
A light singlet at the LHC and DM
}

\section{Jan Kalinowski*}

Faculty of Physics, University of Warsaw, ul. Pasteura 5, 02-093 Warsaw, Poland

E-mail: jan.kalinowski@fuw.edu.pl

An interesting scenario of an R-symmetric supersymmetric model with a light singlet is discussed. Since a light scalar in this model necessarily implies a light Dirac neutralino, its viability as a dark matter candidate is addressed.

Corfu Summer Institute 2017 'School and Workshops on Elementary Particle Physics and Gravity' 2-28 September 2017

Corfu, Greece

${ }^{*}$ Dedicated to the memory of Maria Krawczyk 


\section{Motivation}

The $\mathrm{N}=1$ supersymmetry algebra contains a single global $\mathrm{U}(1)_{R}$ symmetry, called R-symmetry $[1,2]$, with $\left[R, Q_{\alpha}\right]=-Q_{\alpha}$, which implies that component fields of a superfield have R-charges differing by one unit. R-symmetry is stronger than R-parity: since gauge vector fields carry no Rcharge $(R=0)$, gauginos have $\mathrm{R}$-charge $=1$ and therefore, unlike in the Minimal Supersymmetric Standard Model (MSSM), Majorana gaugino mass terms in the soft SUSY breaking potential have $R=2$ and thus are inconsistent with R-symmetry. Gaugino mass terms of Dirac type can be introduced by enforcing an $\mathrm{N}=2$ supersymmetric structure of the gauge/gaugino sector, i.e. adding for each gauge group factor a chiral superfield in the adjoint representation. An exciting implication of R-symmetry is that such a construction necessarily introduces additional scalar fields. This is an interesting option since after the discovery of a Standard Model (SM)-like Higgs boson at the LHC [3, 4, 5], it remains an open question whether there are additional scalar particles, possibly even with smaller mass. Moreover, since R-symmetry forbids also Higgsino mass parameter $\mu$ and all left-right sfermion mixings, alleviating some CP- and flavour-violating constraints [6] and reducing production cross section for squarks making squarks below the $\mathrm{TeV}$ scale generically compatible with LHC data, such a scenario is worth exploring.

\section{Introduction}

Supersymmetric extensions of the SM always predict additional scalars and scenarios with light scalars have been explored both in the minimal supersymmetric standard model (MSSM) and in its extensions, such as the NMSSM which contains a gauge singlet field. While in the MSSM a light scalar is not particularly motivated, in the NMSSM a light singlet-like scalar pushes the tree-level value of the SM-like Higgs boson mass up towards reducing fine-tuning (see e.g. [7] and [8] and references therein for the MSSM and NMSSM, respectively). It turns out that in the minimal R-symmetric supersymmetric model (MRSSM) a scenario with a light scalar is even more motivated since it is necessarily connected with a very light neutralino [9] which can play a role of a dark matter particle.

The MRSSM is defined by the superpotential [10]

$$
\begin{aligned}
W= & \mu_{d} \hat{R}_{d} \cdot \hat{H}_{d}+\mu_{u} \hat{R}_{u} \cdot \hat{H}_{u}+\Lambda_{d} \hat{R}_{d} \cdot \hat{T} \hat{H}_{d}+\Lambda_{u} \hat{R}_{u} \cdot \hat{T} \hat{H}_{u} \\
& +\lambda_{d} \hat{S} \hat{R}_{d} \cdot \hat{H}_{d}+\lambda_{u} \hat{S} \hat{R}_{u} \cdot \hat{H}_{u}-Y_{d} \hat{d} \hat{q} \cdot \hat{H}_{d}-Y_{e} \hat{e} \hat{l} \cdot \hat{H}_{d}+Y_{u} \hat{u} \hat{q} \cdot \hat{H}_{u},
\end{aligned}
$$

where the MSSM-like fields are the Higgs doublet superfields $\hat{H}_{d, u}$ and the quark and lepton superfields $\hat{q}, \hat{u}, \hat{d}, \hat{l}, \hat{e}$. The new fields are the singlet, the $\mathrm{SU}(2)$-triplet and the color-octet chiral superfields, $\hat{S}, \hat{T}$ and $\hat{O}$, which contain the Dirac-mass partners of the usual gauginos. Since the MSSM-like Higgs $\hat{H}_{d, u}$ are assumed to have $R=0$ in the MRSSM, new doublets $\hat{R}_{d, u}$ with $R=2$ are introduced (so called R-Higgs), which contain the Dirac-mass partners of the higgsinos, and the corresponding Dirac-higgsino mass parameters $\mu_{d, u}$. In addition to the standard Yukawa couplings the superpotential contains also Yukawa-like trilinear terms involving the new fields with $\lambda_{d, u}$ parameters for the terms involving the singlet $\hat{S}$ and $\Lambda_{d, u}$ for the terms involving the triplet $\hat{T}$; terms involving the octet $\hat{O}$ are forbidden by R-symmetry. Other parameters of the MRSSM are the Dirac mass parameters $M_{B, W, O}^{D}$ for the $\mathrm{U}(1), \mathrm{SU}(2)_{L}$ and $\mathrm{SU}(3)_{c}$ MSSM-like gauginos $\tilde{B}, \tilde{W}^{a}$ 
and $\tilde{g}^{a}$, which are paired with fermionic components of $\hat{S}, \hat{T}$ and $\hat{O}$ respectively, the soft scalar mass parameters $m_{S, T, O}^{2}$ and $m_{H_{d}, H_{u}, R_{d}, R_{u}}^{2}$ for the singlet, triplet, octet and for the Higgs and R-Higgs states, and the standard $B_{\mu}$ parameter and sfermion mass parameters, while the trilinear sfermion couplings are forbidden by R-symmetry. For the explicit form of the soft SUSY breaking potential see e.g. Ref. [11], and for the detailed discussion of numerical tools used for results presented in this report we refer to [9].

\section{The MRSSM with a light singlet}

In the MRSSM the lightest Higgs boson tree-level mass is typically reduced compared to the MSSM due to mixing with the additional scalars and the loop corrections cannot be enhanced by stop mixing. However, the new fields and couplings give rise to the necessary large loop contributions to the Higgs mass without generating too large a contribution to the W-boson mass, as shown in Refs. [11, 12] where the lightest (SM-like) Higgs boson mass has been computed at the one-loop and leading two-loop level. If on the other hand the SM-like Higgs is assumed to be the secondlightest, the mixing with additional scalars rises its tree-level mass and thus lowers the necessary loop corrections, similarly to what occurs in the NMSSM.

However, unlike in the NMSSM, requiring the second-lightest scalar to be the SM-like imposes constraints on bino-singlino and higgsino masses and couplings. To see this qualitatively, let us consider the neutral Higgs sector of the MRSSM. The real components of neutral scalar fields $\phi_{d}, \phi_{u}, \phi_{S}, \phi_{T}$ of the two MSSM-like Higgs doublets $H_{d, u}$ and the $N=2$ scalar superpartners of the singlet-triplet gauge fields $S$ and $T$ do not mix with the corresponding imaginary components for real vacuum expectation values $v_{d, u, S, T}$. Therefore the full $8 \times 8$ mass-squared matrix breaks into two $4 \times 4$ sub-matrices. In the imaginary component sector the MSSM-like states do not mix with the singlet-triplet states and the mass-squared matrix breaks further into two $2 \times 2$ sub-matrices. Thus the neutral Goldstone boson and one of the pseudo-scalar Higgs bosons $A$ with $m_{A}^{2}=2 B_{\mu} / \sin 2 \beta$ appear as in the MSSM. In the real component sector all four states mix. However, the SM-like Higgs boson is dominantly given by the up-type field $\phi_{u}$ for high $m_{A}$ and $\tan \beta=v_{u} / v_{d}$. In this limit it is enough to consider the $2 \times 2$ sub-matrix of the neutral scalar mass matrix corresponding to the $\left(\phi_{u}, \phi_{S}\right)$ fields only, which reads

$$
\mathscr{M}_{u, S}^{\phi}=\left(\begin{array}{cc}
m_{Z}^{2}+\Delta m_{r a d}^{2} & v_{u}\left(\sqrt{2} \lambda_{u} \mu_{u}^{\mathrm{eff},-}+g_{1} M_{B}^{D}\right) \\
v_{u}\left(\sqrt{2} \lambda_{u} \mu_{u}^{\mathrm{eff},-}+g_{1} M_{B}^{D}\right) & 4\left(M_{B}^{D}\right)^{2}+m_{S}^{2}+\frac{\lambda_{u}^{2} v_{u}^{2}}{2}
\end{array}\right),
$$

where the dominant radiative correction to the diagonal component $\phi_{u}$ is denoted by $\Delta m_{r a d}^{2}, g_{1}$ is the $\mathrm{U}(1)$ gauge coupling and $\mu_{u}^{\text {eff,- }}$ stands for

$$
\mu_{u}^{\mathrm{eff},-}=\mu_{u}+\frac{\lambda_{u} v_{S}}{\sqrt{2}}-\frac{\Lambda_{u} v_{T}}{2} .
$$

The parameters $M_{B}^{D}, \mu_{u}$ and $\lambda_{u}$ appear in the scalar potential due to the usual D-term generation of Dirac mass terms for the MRSSM.

From this approximation it is evident that the light-singlet scenario, in which $m_{H_{1}}<m_{H_{2}} \approx 125$ $\mathrm{GeV}$, can be achieved if the singlet-diagonal element in Eq. (3.1) is smaller than the doublet- 

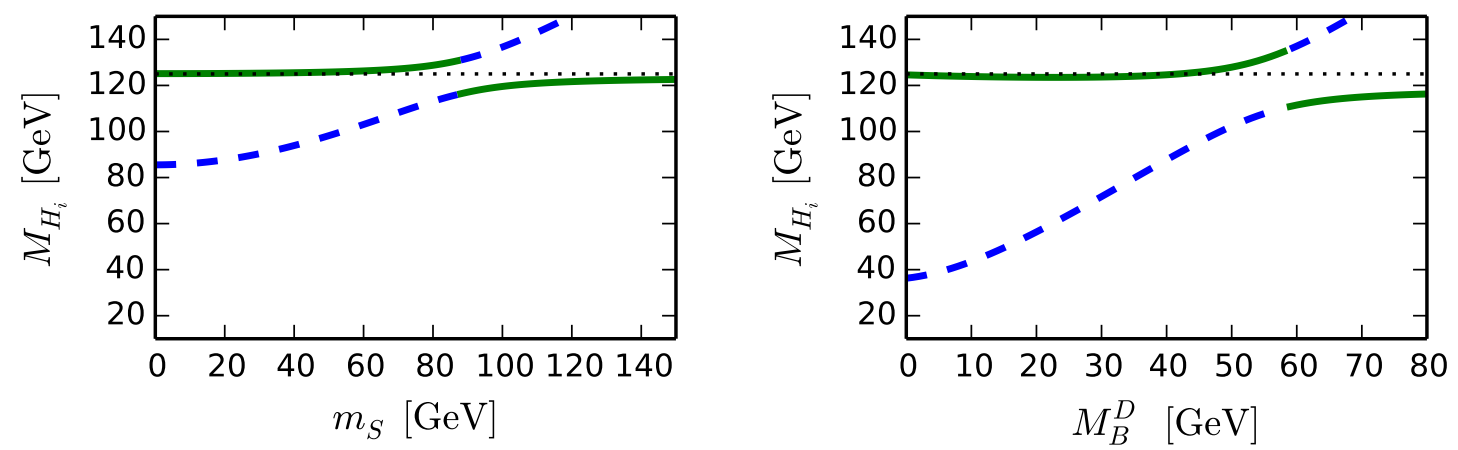

Figure 1: Lightest and second-lightest CP-even Higgs states as functions of the dimensionful parameter $m_{S}$ $\left(M_{B}^{D}\right)$ on the left (right); other parameters are as for BMP5 of Ref. [9]. The green full line indicates that the given state is more SM-like, while the blue dashed line when it is singlet-like.

diagonal one, and off-diagonal elements small enough to avoid tachyonic states. From this requirement one can draw the following hierarchy

$$
m_{S}, M_{B}^{D}<m_{Z}<\mu_{u}, \quad\left|\lambda_{u}\right| \ll 1 .
$$

It implies that not only $m_{S}$ but also the bino-singlino mass parameter $M_{B}^{D}$ must be of order tens $\mathrm{GeV}$, a feature which is unique to the MRSSM.

A quantitative analysis of the masses of the two lightest Higgs states computed to two-loops are shown in Fig. 1 as a function of the two relevant parameters $m_{S}$ (left panel) and $M_{B}^{D}$ (right panel). The non-varying parameters are fixed to the benchmark point BMP5 of Ref. [9], see Appendix for masses of some SUSY particles. If the approximate inequality Eq. (3.3) is satisfied, the lightest state is significantly lighter than $m_{Z}$ and has a high singlet component. When $m_{S}$ or $M_{B}^{D}$ become heavier, the lightest state becomes mainly a doublet-like and hence SM-like. In the light-singlet case, the upward shift of the SM-like Higgs can amount to more than $10 \mathrm{GeV}$, particularly due to the non-vanishing bino-singlino Dirac mass $M_{B}^{D}$, since this parameter also appears in the off-diagonal element of the mass matrix Eq. (3.1).

The novel feature of the light-singlet scenario in the MRSSM is the upper bound on the Dirac bino-singlino mass $M_{B}^{D}$, which has no counterpart e.g. in the NMSSM. Obviously this bound affects the neutralino sector and with LEP bounds on chargino and neutralino production it suggests that the LSP is a Dirac bino-singlino neutralino with mass related to $M_{B}^{D}$ and thus limited from above.

\section{Light-singlet scenario at the LHC}

Experimental data impose direct constraints on the two lightest scalars and their mixing. First, there must be a SM-like state observed at the LHC, with mass and couplings to agree with the observed Higgs signal strengths and branching ratios. Second, the state lighter than the SM-like one has to be mostly singlet-like to pass limits from direct searches for light scalars, especially the LEP searches. Both constraints have been analyzed with HiggsBounds-4.2 and HiggsSignals1.4 [13] and the resulting excluded and allowed regions in the plane of $m_{S}$ and $M_{B}^{D}$ are shown in 

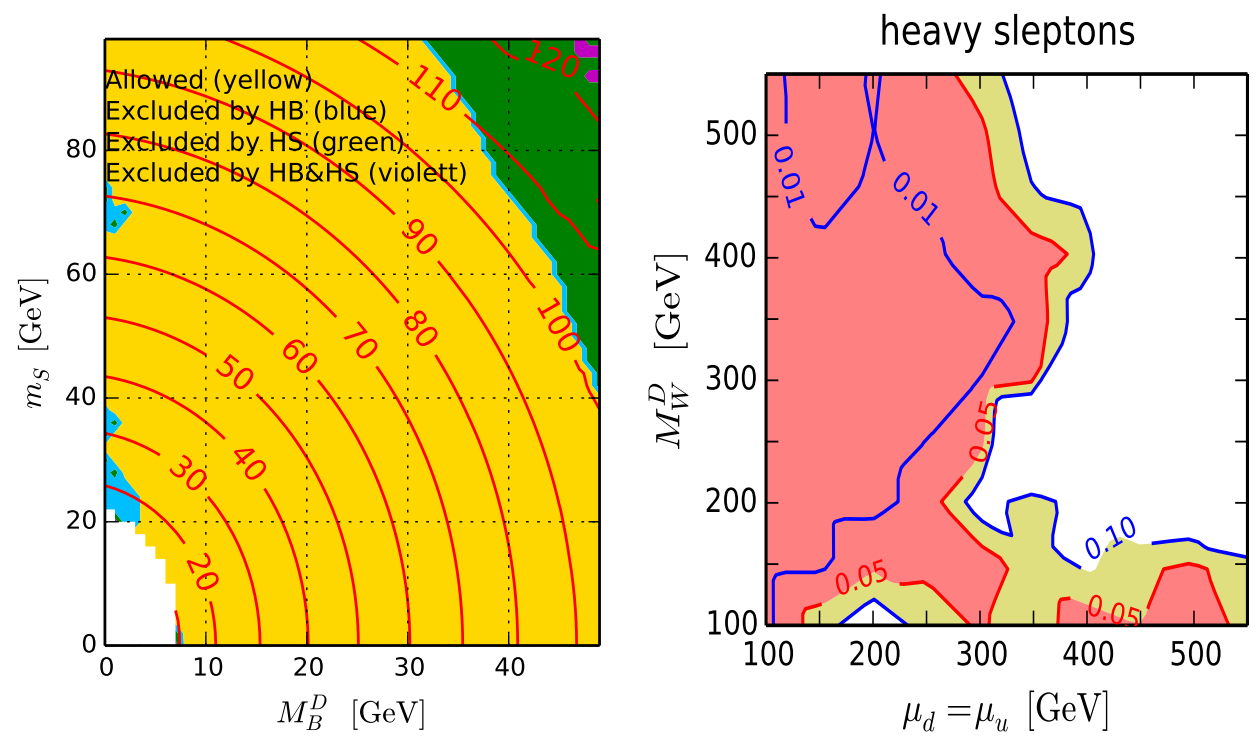

Figure 2: Left: exclusion plot using HiggsSignals-1.3 and HiggsBounds -4.2 with $\Lambda_{u}$ scanned within the range $-1.5<\Lambda_{u}<0$. Red contours show the lightest scalar mass calculated at two loops. Right: exclusion limits for for heavy sleptons as a function of the two higgsino mass parameters $\mu_{d}=\mu_{u}$ and winotriplino mass $M_{W}^{D}$. The red (yellow) region marks the 95\% (90\%) excluded parameter. All other parameters are fixed to the values of BMP5.

the left panel of Fig. 2. The parameter $\Lambda_{u}$ is adjusted to ensure the correct mass of the observed Higgs boson; the remaining parameters are fixed as in BMP5 with $\lambda_{u}=-0.01$. We find that the light-singlet scenario can be realized in the MRSSM for $m_{S}<100 \mathrm{GeV}$ and $M_{B}^{D}<55 \mathrm{GeV}$.

A light bino-like neutralino, and more generally light weakly interacting particles, are interesting since they might lead to observable signals at the LHC and explain the observed dark matter relic density. On the other hand, reinterpreting the LHC bounds in the context of the MRSSM has to be done with care, since the MRSSM differs from the MSSM in the number of degrees of freedom, different mixing patterns, Dirac-versus-Majorana nature of neutralinos, constraints due to the conserved R-charge etc.

In the slepton sector the left-right mixing vanishes in the MRSSM. This is not an essential difference to the MSSM, where typically the mixing is assumed to be small. Since in both models light sleptons decay to leptons and bino-like neutralino LSP, the Dirac or Majorana nature of the LSP is inessential and the MSSM exclusion bounds for light sleptons can be applied to the MRSSM case as well. The chargino and neutralino sectors are quite different. In the MRSSM, the four neutralinos are Dirac fermions composed of eight Weyl spinors $\left(\tilde{B}, \tilde{W}^{0}, \tilde{R}_{d}^{0}, \tilde{R}_{u}^{0}\right)$ and $\left(\tilde{S}, \tilde{T}^{0}, \tilde{H}_{d}^{0}, \tilde{H}_{u}^{0}\right)$ with mass eigenvalues dominantly given by the four independent mass parameters $\left(M_{B}^{D}, M_{W}^{D}, \mu_{d}, \mu_{u}\right)$. There are also four different charginos, with masses determined by the wino and the two higgsino mass parameters. In the MSSM there are only two charginos and the neutralinos are Majorana with only a single higgsino mass parameter $\mu$, so that two neutralino masses are approximately degenerate. Further differences between MRSSM and MSSM exist in the couplings of charginos and neutralinos and therefore in the decay branching ratios. Fixing the bino-singlino to be very 

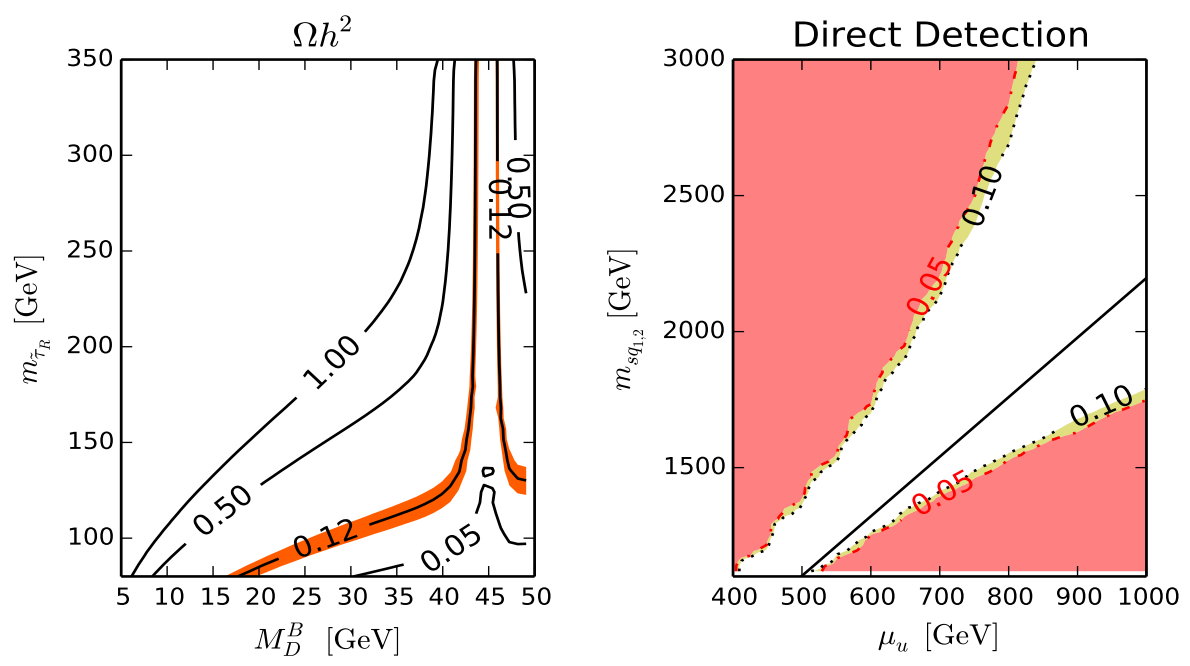

Figure 3: Left panel: dark matter relic density as a function of $M_{D}^{B}$ and $m_{\tilde{\tau}_{R}}$. Right panel: exclusion limits from direct detection dending on $\mu_{u}$ and equal first and second generation squark masses; the straight line shows the relation for the full destructive interference in Eq. (5.2).

light, $\sim 50 \mathrm{GeV}$, there are three relevant mass parameters: the Dirac wino-triplino mass $M_{W}^{D}$ and the two higgsino masses $\mu_{d, u}$. Further, the exclusion limits depend on the slepton masses; for the electroweak and dark matter phenomenology all strongly interacting particles are not relevant and are assumed to be heavy enough to evade limits.

As an illustration we consider a specific case with heavy sleptons which is of interest for dark matter in the light-singlet scenario; for other parameter choices we refer to [9]. Right panel of Fig. 2 shows the exclusion regions in the $\mu_{d}=\mu_{u}-M_{W}^{D}$ plane. Once both higgsinos are heavier than around $300 \mathrm{GeV}$ there is a region with $M_{W}^{D} \geq 200 \mathrm{GeV}$ that is not excluded. The benchmark point MBP5 lies in this viable parameter range.

\section{Light-singlet scenario and dark matter}

Demanding a light singlet Higgs state leads to an upper bound on the bino-singlino mass parameter $M_{B}^{D}<55 \mathrm{GeV}$. In this case the lightest neutralino is bino-singlino dominated, becomes the LSP and it is the dark matter candidate of our model.

For the DM relic density the crucial requirement, as in the MSSM, is to achieve sufficiently effective LSP pair annihilation processes. It turns out that two cases are promising: (a) $m_{\chi_{1}} \approx M_{Z} / 2$ and S-channel resonant LSP pair annihilation into $\mathrm{Z}$ bosons is possible, or (b) right-handed staus are light and annihilation via t-channel stau exchange into tau leptons dominates.

Left panel of Fig. 3 shows the allowed contour in the $M_{B}^{D}-m_{\tilde{\tau}_{R}}$ parameter space. It clearly shows that the measured value of the relic density can be met in two different ways mentioned above: at the $Z$ resonance or away from it. A sharp resonance-like peak is around $M_{B}^{D} \approx M_{Z} / 2$ which results from the S-channel annihilation process, where the required stau mass has to be rather high. This is realized in the benchmark point BMP5. For $M_{B}^{D}$ away from the resonance the required stau mass is below $150 \mathrm{GeV}$, as in benchmark points BMP4 and BMP6 of [9]. 
For the direct DM searches, the spin-independent DM-nucleon scattering cross section at zero momentum transfer can be written in terms of two scattering amplitudes $f_{p}, f_{n}$ as

$$
\sigma_{D M-N}=\frac{4 \mu_{Z_{A}}^{2}}{\pi}\left(Z f_{p}+(A-Z) f_{n}\right)^{2} .
$$

Here $\mu_{Z_{A}}^{2}$ is the dark matter-nucleon reduced mass, and $A$ and $Z$ are atomic mass and number, respectively. It has been noted in Ref. [14] that the spin-independent cross-section for Dirac neutralinos is dominated by the vector part of the $\mathrm{Z}$ boson-exchange and squark-exchange contributions which can lead to large scattering rates and thus to strong bounds on the parameter space.

Since the $\mathrm{Z}$ boson only couples to the (R-)higgsino content of the LSP the corresponding amplitude can be suppressed if the higgsino mass parameters $\mu_{u}$ and $\mu_{d}$ are sufficiently large. Similarly, for the squark-mediated amplitudes squarks need to be also sufficiently heavy.

The right panel of Fig. 3 shows the $95 \%$ and $90 \%$ exclusion bounds (violet (dark) and yellow (light dark) regions) derived using the log likelihood for the direct detection by LUX depending on $\mu_{u}$ and the first/second generation squark masses. The derived limits are quite sensitive to the combination of both parameters. The funnel-shaped allowed region can be qualitatively explained by noting a complete destructive interference between the $\mathrm{Z}$ - and squark-exchange contributions in the limit of degenerate heavy squarks and heavy higgsinos, i.e. $\sigma_{D M-N}=0$, when

$$
m_{\tilde{q}}^{2}=\frac{1}{3}\left(7+11 \frac{A}{Z-A}\right) \mu_{u}^{2} \stackrel{\mathrm{Xe}}{\approx} 4.8 \mu_{u}^{2},
$$

where the numerical value is for Xenon with $A=54$ and $Z=131.3$. The straight line in Fig. 3 (right) corresponds to Eq. (5.2). Away from the line either the squark-mediated or the Z-mediated amplitude becomes small, the destructive interference does not work and experimental limit is not met. Note that the result for the exclusion bounds is calculated using the complete information of micromeGAs [15] and LUXCalc [16], while Eq. (5.2) is only approximated. As the squark masses of the first two generations are limited by LHC searches, the direct detection non-discovery provides a lower limit on $\mu_{u}$. 

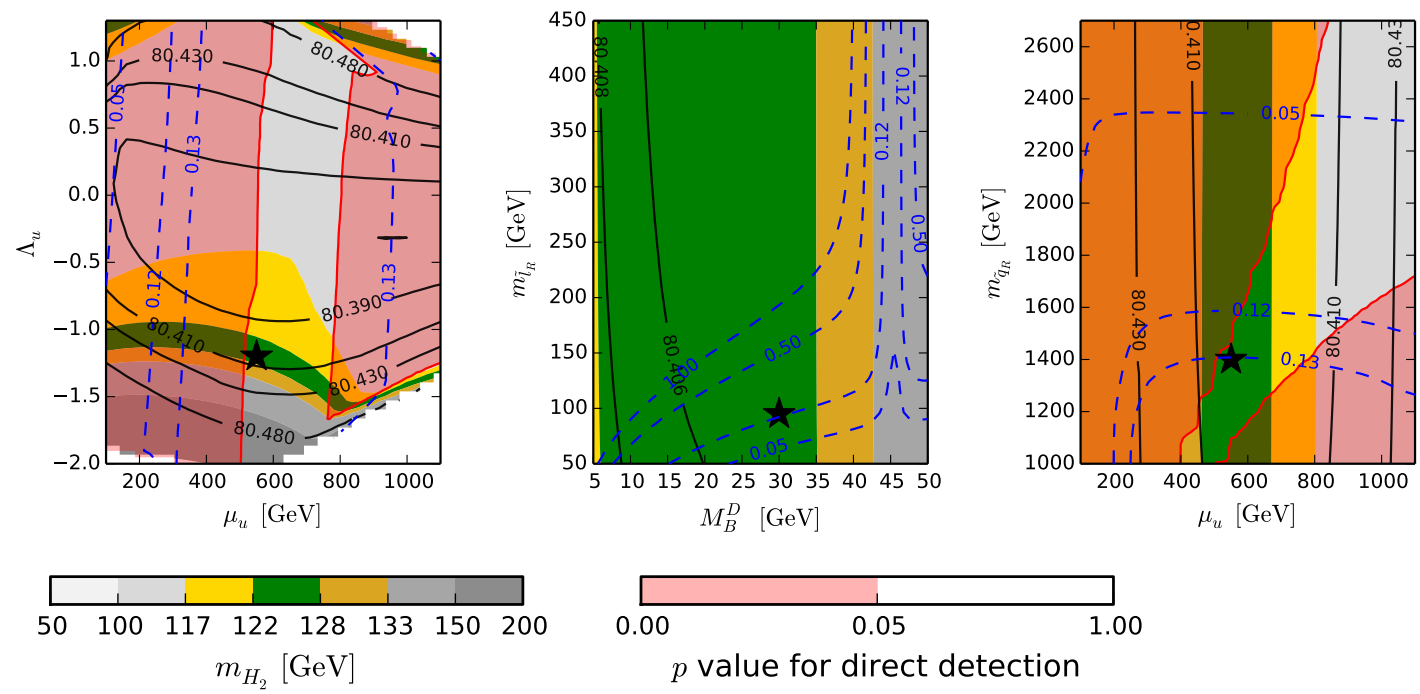

Figure 4: Parameter regions contrasted with experimental constraints. Red areas are excluded with 95\% CL by dark matter direct detection, black full (blue dashed) lines show $m_{W}\left(\Omega h^{2}\right)$. The mass of the SM-like Higgs boson $m_{\mathrm{H}_{2}}$ is given by the colour scale shown. All non-varied parameters are set to the values of BMP6 and BMP6 position is shown by stars.

\section{Summary and outlook}

The minimal R-symmetric model MRSSM is a promising alternative to the MSSM. In particular, the light-singlet scenario is very attractive since it provides an increased tree-level SM-like Higgs boson mass and many light weakly interacting particles which could be discovered at the next LHC run as well as the possibility to explain dark matter. It is also very predictive as can be inferred from Fig. 4. If a light singlet-like Higgs boson is found, not only the parameter $m_{S}$ but also $M_{B}^{D}$ and $\lambda_{u}$ must be very small. Then, the requirement of the $125 \mathrm{GeV}$ Higgs mass essentially fixes $\Lambda_{u}$ and constrains $M_{B}^{D}$ and $\mu_{u}$ (left panel), implying that the fermionic partner is the LSP. On the other hand dark matter data constrain slepton and squark masses (middle and right panels) and other electroweak parameters pointing to interesting mass hierarchies of SUSY particles to be searched at the LHC. It would also be interesting to explore the ILC discovery potential at its initial $250 \mathrm{GeV}$ phase [17] for the benchmark points discussed here.

\section{Acknowledgments}

I thank Philip Diessner, Wojtek Kotlarski and Dominik Stöckinger for many valuable discussions and fruitful collaboration. Thanks also go to Gudrid Moortgat-Pick for her hospitality during my stay at DESY. Work supported in part by the Polish National Science Centre HARMONIA grant under contract UMO-2015/18/M/ST2/00518 (2016-2019) and the DFG through the SFB 676 "Particles, Strings and the Early Universe". 


\section{Appendix}

Masses of some SUSY particles relevant for the discussion in this report. All values are given in GeV. The four charginos appearing in the MRSSM are denoted as $\chi_{1}^{ \pm}, \chi_{2}^{ \pm}, \rho_{1}^{ \pm}, \rho_{2}^{ \pm}$.

\begin{tabular}{cccccccccccccc}
\hline & $\chi_{1}^{0}$ & $\chi_{2}^{0}$ & $\chi_{3}^{0}$ & $\chi_{4}^{0}$ & $\chi_{1}^{ \pm}$ & $\chi_{2}^{ \pm}$ & $\rho_{1}^{ \pm}$ & $\rho_{2}^{ \pm}$ & $\tilde{\tau}_{R}$ & $\tilde{\mu}_{R}$ & $\tilde{e}_{R}$ & $\tilde{\ell}_{L}$ & $m_{H_{1}}$ \\
\hline BMP4 & 49.8 & 132 & 617 & 691 & 131 & 625 & 614 & 713 & 128 & 802 & 802 & 808 & 100 \\
BMP5 & 43.9 & 401 & 519 & 589 & 409 & 524 & 519 & 610 & 1000 & 1001 & 1001 & 1005 & 94 \\
BMP6 & 29.7 & 427 & 562 & 579 & 422 & 562 & 433 & 587 & 106 & 353 & 353 & 508 & 95 \\
\hline
\end{tabular}

\section{References}

[1] A. Salam and J. A. Strathdee, Nucl. Phys. B 87 (1975) 85.

[2] P. Fayet, Nucl. Phys. B 90 (1975) 104.

[3] G. Aad et al. [ATLAS Collaboration], Phys. Lett. B 716 (2012) 1.

[4] S. Chatrchyan et al. [CMS Collaboration], Phys. Lett. B 716 (2012) 30.

[5] The ATLAS and CMS Collaborations, ATLAS-CONF-2015-044.

[6] G. D. Kribs and A. Martin, Phys. Rev. D 85 (2012) 115014.

[7] P. Bechtle et al., Eur. Phys. J. C 77 (2017) 67.

[8] U. Ellwanger and M. Rodriguez-Vazquez, JHEP 1602 (2016) 096.

[9] P. Diessner, J. Kalinowski, W. Kotlarski and D. Stöckinger, JHEP 1603 (2016) 007.

[10] G. D. Kribs, E. Poppitz and N. Weiner, Phys. Rev. D 78 (2008) 055010.

[11] P. Diessner, J. Kalinowski, W. Kotlarski and D. Stöckinger, JHEP 1412 (2014) 124.

[12] P. Diessner, J. Kalinowski, W. Kotlarski and D. Stöckinger, Adv. High Energy Phys. 2015 (2015) 760729.

[13] P. Bechtle, O. Brein, S. Heinemeyer, G. Weiglein and K. E. Williams, Comput. Phys. Commun. 181 (2010) 138; ibid. 182 (2011) 2605.

P. Bechtle et al., PoS CHARGED 2012 (2012) 024; Eur. Phys. J. C 74 (2014) 2693.

P. Bechtle, S. Heinemeyer, O. Stal, T. Stefaniak and G. Weiglein, Eur. Phys. J. C 75 (2015) 421; ibid. 74 (2014) 2711.

[14] M. R. Buckley, D. Hooper and J. Kumar, Phys. Rev. D 88 (2013) 063532.

[15] G. Belanger, F. Boudjema, A. Pukhov and A. Semenov, Comput. Phys. Commun. 192 (2015) 322.

[16] C. Savage, A. Scaffidi, M. White and A. G. Williams, Phys. Rev. D 92 (2015) 103519.

[17] S. Asai et al., arXiv:1710.08639 [hep-ex]. 